\title{
Markus Hoppe*
}

\section{Kommunale Arbeitgeberverbände und die Privatisierung öffentlicher Dienste: Auswirkungen auf Verbandsstruktur und -politik}

\section{Zusammenfassung}

Kommunale Arbeitgeberverbände vertreten als Tarifakteure die Arbeitgeberinteressen der Kommunen und ihrer Unternehmen. Der Strukturwandel des öffentlichen Sektors seit den 1990er Jahren führte auch in ihrem Organisationsbereich zu tiefgreifenden Veränderungen, deren Dynamik von der Liberalisierung der Produkt- und Dienstleistungsmärkte ausging und vielfach zu Privatisierungen und Ausgründungen führte. Der Beitrag diskutiert, wie sich kommunale Arbeitgeberverbände auf die veränderten Wettbewerbsbedingungen eingestellt haben. In den Blick kommen sowohl Anpassungen der Verbandsstruktur als auch Veränderungen des verbandspolitischen Outputs. Im Ergebnis zeigt sich zumeist eine der Mitgliedschaftslogik folgende Handlungsorientierung, wobei der Fokus auf der Rolle als Tarifvertragspartei liegt.

Schlagwörter: Arbeitgeberverbände, öffentlicher Sektor, Liberalisierung, Tarifpolitik

\section{Municipal Employers' Associations and the Privatisation of Public Services: Impact on Associational Structure and Policy}

\section{Summary}

Municipal employers' associations represent the interests of municipalities and their enterprises in collective bargaining. Since the early 1990s structural changes in the public sector can be observed, these brought about the liberalisation of goods and services markets, specifically the large-scale privatisation and outsourcing of public services. In tune these developments have resulted in profound modifications in employers' organisational domains. This contribution discusses how municipal employers' associations have adapted to these competitive conditions. Here the focus is on how association structures have adjusted as well as changes in the associations' political outputs. These initiatives mostly follow a membership-logic, with specific focus on the collective bargaining actor.

Keywords: Employers' associations, public sector, deregulation, collective bargaining (JEL: J5, L32, J45)

* Markus Hoppe, wissenschaftlicher Mitarbeiter, Universität Tübingen, Forschungsinstitut für Arbeit, Technik und Kultur (FATK), Haußerstraße 43, 72076 Tübingen. E-Mail: markus.hoppe@uni-tuebingen.de

** Artikel eingegangen: 28.07.2016, revidierte Fassung akzeptiert nach doppelt-blindem Begutachtungsverfahren: 15.08 .2017 


\section{Einleitung}

Der öffentliche Sektor der BRD hat sich seit den frühen 1990er Jahren nachhaltig verändert. Zum einen sind die Beschäftigtenzahlen von 6,74 Mio. im Jahr 1991 auf nunmehr 4,19 Mio. im Jahr 2015 deutlich zurückgegangen (Keller 2014a, S. 34; StatBA, 2016). Zum anderen erfuhr auch die Architektur des öffentlichen Sektors einen tiefgreifenden Wandel, indem viele ehemals staatlich organisierte öffentliche Aufgaben zwischenzeitlich in Teilen oder vollständig privatisiert wurden. Der Beitrag analysiert, welche Rolle die kommunalen Arbeitgeberverbände in diesem Prozess des Strukturwandels spielen. Besonderes Augenmerk liegt dabei auf den Auswirkungen von Liberalisierung und Privatisierung auf das Verbandshandeln.

Der sektorale Strukturwandel wird vor allem auf zwei Aspekte zurückgeführt. Es wird konstatiert, dass der öffentliche Dienst beginnend in den 1980er Jahren aufgrund der sich verschlechternden Lage der öffentlichen Haushalte in eine Legitimitätskrise geriet (Hebeler, Sitzer \& Tews, 2012), in deren Folge die öffentliche Dienstleistungserbringung unter Kostenaspekten zunehmend infrage gestellt wurde. Die Dynamik ging dabei von den Liberalisierungs- und Privatisierungsbewegungen aus, die als Ausweg aus der angespannten öffentlichen Haushaltslage galten. Öffentliche Dienste wurden für marktgängig erklärt (Mühlenkamp, 2003), der privaten Dienstleistungserbringung wurden Effektivitäts- und Effizienzvorteile vorausgesagt und der Staat wurde normativ auf die Rolle des Regulierungsoder Gewährleistungsstaates reduziert (Engartner, 2008). „Als Folge dieser Entwicklung wurde der öffentliche Dienst mehr als Kostenfaktor denn als Instrument zur sachadäquaten Lösung gesellschaftlicher Probleme gesehen“ (Schneider \& Simon, 2014, S. 86). Damit fand auch eine Umdeutung administrativer Probleme in ökonomische statt, die nur ökonomisch zu lösen seien (Czerwick, 2008, S. 68). Resultat war eine „Verschlankung“ des öffentlichen Dienstes, die in Deutschland besonders weit ging. Im OECD-Vergleich gilt der öffentliche Sektor der BRD als relativ klein (Bosch, Mesaros, Schilling \& Weinkopf, 2012, S. 11). Unabhängig von der konkreten Form folgt aus der Privatisierung die Durchdringung der öffentlich verantworteten Dienstleistungserbringung mit privatem Kapital (Bosch, 2013). Auf der betrieblichen Ebene setzte sich das Ökonomisierungsprogramm mit der Umsetzung des Neuen Steuerungsmodells (KGSt, 1993) fort, das sich resümierend jedoch nicht als „Allheilmittel“ (Bogumil, 2014, S. 55) erwies. So kommen auch aktuelle Untersuchungen zur Rolle des Staates als Arbeitgeber zu dem Schluss, dass sich der Druck auf die öffentliche Dienstleistungserbringung infolge knapper Ressourcen in demokratischen Gesellschaften nur über den Wählerwillen entladen kann, indem demokratisch legitimierte Gegenentwürfe mehrheitsfähig werden (Gottschall, Kittel, Briken, Heuer, Hils, Streb \& Tepe, 2015). Neben der Privatisierung führte der Beitritt der ehemaligen DDR zur Bundesrepublik Deutschland zu sektoralen Strukturänderungen, da insbesondere die Angleichung des öffentlichen Dienstes der neuen Bundesländer an das westdeutsche Modell zu bewältigen war (Keller \& Henneberger, 1992). Dies hatte weitreichende Folgen für die öffentliche Beschäftigung, indem Veränderungen in der Zusammensetzung der Gebietskörperschaften ebenso zur Personalreduzierung führten wie die Entbürokratisierung des als ,aufgebläht“ geltenden Verwaltungsapparats der ehemaligen DDR.

Der Beitrag ist wie folgt gegliedert: In Abschnitt 2 wird der Forschungsstand über kommunale Arbeitgeberverbände unter den besonderen sektoralen Bedingungen des öffent- 
lichen Dienstes skizziert, auf dessen Basis in Abschnitt 3 die Forschungsfragen abgeleitet und zentrale Hypothesen formuliert werden. Um die Wirkungen der sektoralen Strukturveränderungen auf das Verbandshandeln analysieren zu können, wird im Abschnitt 4 die Funktionsweise kommunaler Arbeitgeberverbände erläutert, indem Aspekte der Arbeitsteilung, der innerverbandlichen Interessenbearbeitung sowie der Willensbildung diskutiert werden. Anschließend geht es in Abschnitt 5 um das Agieren kommunaler Arbeitgeberverbände im sektoralen Strukturwandel, indem die Folgen von Privatisierung und Outsourcing sowie das Auftreten neuer Verhandlungspartner auf Gewerkschaftsseite für ihr Handeln dargelegt werden. In Abschnitt 6 werden die Reaktionen auf die beschriebenen strukturellen Änderungen im Verbandshandeln dargestellt, bevor in Abschnitt 7 ein Fazit im Hinblick auf die einleitend formulierte Fragestellung gezogen wird.

Die Analyse stützt sich empirisch auf qualitative Experteninterviews (Gläser \& Laudel, 2010), die als teilstandardisierte, problemzentrierte Leitfadeninterviews (Lamnek, 2010) mit zehn haupt- und ehrenamtlichen Verbandsvertreterinnen und -vertretern in drei kommunalen Arbeitgeberverbänden geführt wurden. Einbezogen wurde der Dachverband Vereinigung der kommunalen Arbeitgeberverbände (VKA) sowie zwei regionale kommunale Arbeitgeberverbände (KAV) unterschiedlicher Größe, wobei überwiegend hauptamtliche Akteure für die Gespräche gewonnen werden konnten (Verbandsgeschäftsführer, Referentinnen und Referenten) und lediglich bei einem Verband ein Interview auf ehrenamtlicher Ebene möglich war. Das Interviewmaterial wurde vollständig transkribiert und inhaltlich strukturiert (Mayring, 2015). Ergänzend wurden Dokumente (Jahresberichte, Pressemitteilungen, offizielle Verlautbarungen) analysiert und statistische Informationen (etwa zur Mitgliederstruktur und -entwicklung) aufbereitet.

\section{Forschungsstand}

Das Verbandshandeln von Arbeitgeberorganisationen wurde in mehrerlei Hinsicht zu erklären versucht. Dem Paradigma des methodologischen Individualismus folgend geht der Ansatz der „Logik des kollektiven Handelns“ (Olson, 2004) davon aus, dass sich Arbeitgeberverbände nur dann bilden, wenn sie als Resultat rationaler Entscheidungen einzelner Unternehmen Kollektivgüter bereitstellen können, etwa die abgeschlossenen Tarifverträge und die mit ihrer Anwendung verbundene Friedenspflicht, einhergehend mit dem Wegfall der Kosten für die individuelle Aushandlung der Beschäftigungsbedingungen. Erschwert würde die Verbandsbildung jedoch dadurch, dass rational handelnde Akteure bzw. Unternehmen auch dann von den kollektiven Gütern profitieren, wenn sie sich selbst als Trittbrettfahrer nicht an den Kosten ihrer Herstellung beteiligen (free rider-Problem). Daher müssten Verbände zusätzlich ,,selektive Anreize“" setzen, deren Inanspruchnahme ausschließlich den Mitgliedern vorbehalten ist. So sieht Olson in der Koppelung selektiver Anreize mit Kollektivgütern das Motiv für den Verbandsbeitritt, indem „kollektive Güter als Nebenprodukte der selektiven Anreizsetzung“ (Henneberger, 2017, S. 176) entstehen. Verbände erscheinen in dieser Perspektive als „Instrumente zur rationalen Aggregation von Interessen“ (Behrens, 2013, S. 60).

Entgegen dieser Auffassung betonen Vertreter des Neokorporatismus die Rolle von Kollektivakteuren und die unterstützende Rolle des Staates bei der Organisierung von Inte- 
ressen. Kollektives Handeln als Ausdruck einer Harmonisierung unterschiedlicher Ausgangsinteressen unter Berücksichtigung des staatlichen Einflusses wird in dieser Perspektive durch die gleichzeitige Nutzung zweier Handlungslogiken möglich, einer an den Mitgliederinteressen orientierten Mitgliedschaftslogik (logic of membership) und einer an der Umwelt orientierten Einflusslogik (logic of influence) (Schmitter \& Streeck, 1981).

Gelegentlich wird auch Traxlers Ressourcenansatz hervorgehoben (Behrens, 2013, S. 61), demnach Unternehmen in der Regel über genügend Ressourcen verfügen, um ihre Eigeninteressen auch im Alleingang zu verfolgen, sollten die Ziele ihrer Verbände davon abweichen. Daher seien Verbände gut beraten, ,ihre Politik möglichst weitgehend den Eigenund Sonderinteressen spezifischer Mitgliedergruppen anzupassen“ (Traxler, 1993, S. 159). Unternehmerverbände gelten als „kleine Gruppen“ im Sinne Olsons und sind als solche in hohem Maße organisationsfähig, sie haben aber gleichsam die Herausforderung zu bewältigen, die eigenmächtigen Mitglieder auf die Verbandspolitik zu verpflichten. Nicht wenige Arbeitgeberverbände tragen diesem Paradox durch ein an die Betriebsgröße gekoppeltes Stimmrecht in den politischen Verbandsgremien Rechnung, um die Mitglieder von der Nutzung der „Exit““-Option der Abwanderung (Hirschman, 1974) abzuhalten. Ein nach der Beschäftigtenzahl differenziertes Stimmrecht in der Mitgliederversammlung ist auch für kommunale Arbeitgeberverbände typisch.

Fokussiert auf die Arbeitsbeziehungen des öffentlichen Sektors offenbart ein Blick in die Literatur, dass diese sich aufgrund von besonderen ,rechtlich-institutionellen Rahmenbedingungen“ (Keller, 2010, S. 43) von jenen in der Privatwirtschaft unterscheiden. Dazu gehört, dass der Staat in den Arbeitsbeziehungen eine Doppelrolle als Gesetz- und Arbeitgeber gleichermaßen einnimmt (Hyman, 2008). Für die Analyse der Arbeitsbeziehungen wird daraus gefolgert, dass sie nicht ohne Berücksichtigung dieser besonderen Rahmenbedingungen auskommen kann und der Vergleich mit den privatwirtschaftlichen industriellen Beziehungen zu Fehlinterpretationen verleitet (Mehde, 2011). Zudem ist es im öffentlichen Sektor schwieriger zu identifizieren, wer genau Adressat der Arbeitgeberfunktion ist, da man es mit komplexen Verantwortungs- und Entscheidungsstrukturen zu tun hat, die bei der Bereitstellung öffentlicher Güter und Dienstleistungen zumeist verfassungsrechtlich oder politisch vorgegeben sind (Keller, Due \& Anderson, 2001, S. 72).

Hinzu kommt die besondere Personalstruktur des öffentlichen Dienstes, die sich in Deutschland in der Dualität von Tarifbeschäftigten sowie Beamtinnen und Beamten ausdrückt (Hebeler, 2008). Während Letztgenannte sich in einem besonderen Dienst- und Treueverhältnis dem Staat gegenüber befinden, besteht für Tarifbeschäftigte ein privatrechtliches Arbeitsverhältnis. Kommunale Arbeitgeberverbände sind als Tarifakteure nur für Tarifbeschäftigte zuständig, wenngleich durch die institutionelle Verzahnung mit kommunalen Spitzenverbänden, in denen die Kommunen in ihrer Eigenschaft als Dienstherren ebenfalls Mitglied sind, eine wechselseitige Orientierung bei der Festlegung von Beschäftigungsbedingungen beider Statusgruppen angestrebt wird. Traditionell folgte die Beamtenbesoldung der allgemeinen Tarifentwicklung. Seit der Föderalismusreform II im Jahr 2006 liegt die Zuständigkeit für die Beamtenbesoldung jedoch bei den Ländern, was zwischenzeitlich Differenzen in den Besoldungspraktiken bewirkte (Czerwick, 2008). Der Beamtenanteil beträgt im kommunalen öffentlichen Dienst aktuell 12,9 Prozent, was verglichen mit Bund $(55,5 \%)$ und Ländern (54,2\%) ein niedriger Wert ist (StatBA, 2016, eigene Berechnung). 
Für die Analyse kommunaler Arbeitgeberverbände sind des Weiteren die Befunde aus Forschungsarbeiten zu privatwirtschaftlichen Arbeitgeberverbänden von Relevanz. Empirische Studien zeigen, dass Arbeitgeberverbände seit den 1980er Jahren an rückläufigen Mitgliederzahlen leiden und sie deshalb zur Stabilisierung der Organisationsstärke und zur Vermeidung von Verbandsflucht seit den 1990er Jahren verstärkt auf flexible Mitgliedschaftsformen setzen. In der Privatwirtschaft gilt der Rückzug der Arbeitgeber aus der Verbandstarifbindung längst als strategische Option. Arbeitgeberverbände reagierten darauf mit dem Angebot von OT-Mitgliedschaften (Mitgliedschaften ohne Tarifbindung), also der Wahlmöglichkeit zwischen tarifgebundenem und tariffreiem Status innerhalb des Verbandes (Schroeder, 2007). Noch einen Schritt weiter gehen die OT-Verbände, die gänzlich auf Tarifbindung verzichten. „OT-Mitgliedschaften und OT-Verbände lassen sich als eine Antwort der Arbeitgeberverbände auf wachsende Schwächen der eigenen Organisationen verstehen“ (Haipeter, 2017, S. 307). Die strategische Nutzung tariffreier Mitgliedschaften zur Verbandsstabilisierung wird als „Umprogrammierung“ bezeichnet (Behrens, 2011, S. 49) und hat als Ausdruck einer neuen Dominanz der Mitgliedschaftslogik zur Folge, dass Arbeitgeberverbände ,keine Veranlassung mehr [haben], die Tarifpolitik kreativ zu gestalten“" (Rehder, 2009, S. 64).

Bislang wenig erforscht sind die Auswirkungen der sektoralen Besonderheiten für das Handeln der Kollektivakteure im öffentlichen Dienst. Die wenigen existierenden Beiträge haben entweder einen starken analytischen Fokus und kommen ohne empirische Breite aus (Keller, 2017; Keller, 2014b) oder das Erkenntnisinteresse liegt auf der Umsetzung spezifischer Tarifthemen (z.B. leistungsorientierte Bezahlung, Qualifizierung), wodurch das Handeln der Tarifakteure nur durch die Brille des jeweiligen Untersuchungsgegenstands erfasst wird (etwa Schmidt \& Müller, 2013; Bahnmüller \& Hoppe, 2014).

\section{Fragestellungen und Hypothesen}

Vor dem Hintergrund des skizzierten Forschungsstands wird in diesem Beitrag analysiert, inwieweit die sektoralen Strukturveränderungen infolge von Liberalisierung und Privatisierung Auswirkungen auf das Handeln kommunaler Arbeitgeberverbände hatten. Dabei wird insbesondere auf Entwicklungen in der Verbandsstruktur, der innerverbandlichen Interessenbearbeitung und der Verbandspolitik eingegangen.

Infolge der Privatisierung ehemals öffentlicher Dienste agiert ein relevanter Teil der Verbandsmitglieder zunehmend im Wettbewerb und hat folglich einen Bedarf an wettbewerbsermöglichenden tariflichen Rahmenbedingungen. Auf der kommunalen Ebene steht mit der VKA ein „,echter Dachverband“ (Keller, 2010, S. 43) zur Verfügung, dessen Verbandszweck in der Vertretung der ,gemeinsamen Angelegenheiten ihrer Mitglieder und der diesen angeschlossenen Arbeitgeber auf tarif-, arbeits- und sozialrechtlichem Gebiet gegenüber Gewerkschaften, staatlichen Stellen und anderen Organisationen“ (VKA, 2014a, S. 2) besteht. Demnach ist zu erwarten, dass kommunale Arbeitgeberverbände durch die Erfüllung ihres Auftrags mit ihren Tarifverträgen zur Förderung von Wettbewerbsfähigkeit beitragen und dadurch auf den sektoralen Wandel reagieren.

Anzunehmen ist weiterhin, dass die Einbindung kommunaler Arbeitgeber und ihrer Verbände in das Institutionengefüge des öffentlichen Dienstes dazu beiträgt, dass die aus 
der Privatwirtschaft bekannten Mechanismen der Marktkonkurrenz unter den Mitgliedern nicht in vergleichbarer Weise auftreten. Die Tarifpolitik im öffentlichen Sektor wird von einer langen Kette politisch vorgelagerter, sektorspezifischer Entscheidungsstrukturen beeinflusst, etwa in Form der institutionellen Verschränkung mit den kommunalen Spitzenverbänden und der Abstimmung mit übergeordneten staatlichen Ebenen im Rahmen gemeinsamer Tarifverhandlungen mit dem Bund. Dies dürfte auch zur Folge haben, dass im öffentlichen Sektor aufgrund einer komplexeren, über ökonomische Motive hinausgehenden Mitgliedschaftsentscheidung ein höheres Maß an (Mitglieder-) Stabilität vorhanden ist.

Zudem hat die Liberalisierungswelle nicht alle Mitgliedersegmente gleichermaßen ergriffen. Während die Kommunalverwaltungen nach wie vor überwiegend politisch gesteuert werden, wirken in den kommunalwirtschaftlichen Bereichen (Krankenhäuser, Ver- und Entsorgungsbetriebe, Nahverkehrsbetriebe) zunehmend auch Mechanismen der Marktsteuerung. Daher ist anzunehmen, dass die unterschiedliche Betroffenheit von Privatisierungen zu Unterschieden in der innerverbandlichen Interessenbearbeitung zwischen den Mitgliedersegmenten führt.

\section{Kommunale Arbeitgeberverbände als Tarifakteure im öffentlichen Sektor}

\subsection{Verbandsstruktur und Mitglieder}

Die Vertretung der Arbeitgeberinteressen weist Verbandsstrukturen am deutlichsten auf der kommunalen Ebene auf. Dies dürfte vor allem pragmatische Gründe haben, da die (hohe) Anzahl der in den KAV organisierten Arbeitgeber eine verbandliche Interessenorganisation erfordert. ${ }^{1}$ Zudem verfügen die Kommunen als verfassungsrechtlich definierter Teil der Länder über keine „Staatsqualität“ (Rehm \& Matern-Rehm, 2010, S. 25), weshalb eine verbandliche (und keine unmittelbar staatliche) Vertretung der Arbeitgeberinteressen naheliegt.

Die VKA kann als Dachverband und Spitzenorganisation im Sinne des Tarifrechts im Namen ihrer Mitglieder bzw. der von ihnen vertretenen Arbeitgeber Tarifverträge abschließen. In der VKA sind 16 regionale, rechtlich selbstständige und in jedem Bundesland existierende kommunale Arbeitgeberverbände (KAV) organisiert, die ,,autonome Rechte aus dem Kanon ihrer Tarifhoheit an die VKA ab[geben]“ (Keller, 2013, S. 502). Die VKA nimmt „Koordinationsaufgaben“ wahr und ist „für Fragen von allgemein-überregionalem Interesse zuständig“" (ebd.).

In den landesbezirklichen KAV sind dann die einzelnen kommunalen Arbeitgeber organisiert, die sich aus kommunalen Gebietskörperschaften (Städte, Gemeinden, Landkreise) und deren Unternehmen zusammensetzen (Krankenhäuser, Ver- und Entsorgungsbetriebe, Nahverkehrsunternehmen, Häfen und Flughäfen). Hinzu kommen die Sparkassen. Neben den regulären Mitgliedern gibt es in den politischen Gremien auf Dachverbands- und regionaler Ebene noch eine Reihe von ständigen Gästen ohne Stimmrecht. Dazu gehören in erster Linie die kommunalen Spitzenverbände (Deutscher Städtetag, Deutscher Städte- und

1 Die VKA gibt auf ihrer Homepage die Anzahl der bei ihren Mitgliedverbänden organisierten Arbeitgeber mit „über 10.000“ an (www.vka.de). 
Gemeindebund, Deutscher Landkreistag) und die für einzelne Mitgliedersegmente zuständigen Fachverbände in den jeweiligen Gruppenausschüssen. Hierzu zählen etwa die Deutsche Krankenhausgesellschaft (DKG), der Verband Deutscher Verkehrsunternehmen (VDV) oder die Sparkassen- und Giroverbände.

Bis 2006 existierte arbeitgeberseitig eine Verhandlungsunion aus Bund, Ländern und Kommunen, die jedoch von den Ländern bei der Vorbereitung des Tarifvertrags für den öffentlichen Dienst (TVöD), der die bis dahin gültigen Tarifverträge für Arbeiter und Angestellte ersetzen sollte, letztlich aus Kostengründen verlassen wurde (Schmidt, Müller \& Trittel, 2011). Die Verhandlungsgemeinschaft aus Bund und Kommunen besteht aber weiterhin fort, auch wenn sie gelegentlich auf Mitgliederversammlungen der VKA auf den Prüfstand gestellt wurde.

\subsection{Arbeitsteilung und Verbandspolitik}

Die Verbandsarbeit kommunaler Arbeitgeberverbände ist unterteilt in die Geschäftsstellenarbeit und die (verbands-)politische Arbeit. Während erstere vom hauptamtlichen Personal der Geschäftsführungs- und Referentenebene geleistet wird, obliegt die Verbandspolitik den ehrenamtlichen Funktionsbereichen (mit Unterstützung der Geschäftsstellen), die in den VKA-Gremien Mitgliederversammlung, Präsidium und Geschäftsführerkonferenz die Entscheidungsfindung verantworten. Diese Aufgabenverteilung gilt sowohl für die Dachverbands- als auch für die regionale Ebene.

$\mathrm{Zu}$ den Aufgaben der Geschäftsstellen gehört auch die Bereitstellung verbandlicher Serviceleistungen, die nach Olsons Theorie den selektiven Anreizen zuzurechnen sind und insofern als ein Mitgliedschaftsmotiv fungieren. Sie umfassen verschiedene Elemente: Erstens betreibt die VKA einen Rundschreibenservice, mit dem aktuelle Informationen zu arbeits- und tarifrechtlichen Fragen zur Kenntnis gebracht werden. Zwischen 2008 und 2014 wurden monatlich zwischen 25 und 35 Rundschreiben durch die VKA versandt. Die Hauptlast im Mitgliederservice liegt bei den regionalen KAV. Sie übernehmen u.a. die Rechtsberatung sowie die Prozessvertretung einzelner Arbeitgeber vor Arbeitsgerichten. Die Beratung durch das juristisch geschulte KAV-Personal erfolgt im Regelfall telefonisch, in Ausnahmefällen aber auch durch Inhouse-Konsultationen. Darüber hinaus wird der Erfahrungsaustausch zwischen den Arbeitgebern gefördert, indem themenbezogene Arbeitskreise organisiert werden. Zudem bieten die KAV Fortbildungen zu arbeits- und tarifrechtlichen Themen an. Der Mitgliederservice kommunaler ist mit jenem privatwirtschaftlicher Arbeitgeberverbände (Henneberger, 2017, S. 176) vergleichbar.

\subsection{Organisationsdomäne}

Die in der VKA mit Tarifbindung organisierten Arbeitgeber haben derzeit etwa 2,1 Mio. Beschäftigte. Wie Tabelle 1 zeigt, entfällt der größte Anteil auf die Verwaltungen, gefolgt von Krankenhäusern und Pflegeeinrichtungen sowie Sparkassen. Weniger besetzungsstark sind die Ver- und Entsorgungsbetriebe, die Nahverkehrsbetriebe und die Flughäfen. Gerade diese kleineren Bereiche gelten bei kommunalen Arbeitgeberverbänden als sensibel bei Arbeitskämpfen, da sie bedeutsam für die kommunale Infrastruktur und deshalb die Auswirkungen im Streikfall wirksam und öffentlich sichtbar sind. 
Tabelle 1: Personalbestand der Mitglieder der kommunalen Arbeitgeberverbände (in Tausend)

\begin{tabular}{lccccc}
\hline Sparte & $\mathbf{2 0 1 0}$ & $\mathbf{2 0 1 3}$ & $\mathbf{2 0 1 4}$ & $\mathbf{2 0 1 5}$ & $\mathbf{2 0 1 6}$ \\
\hline Verwaltungen & 1100 & 1171 & 1190 & 1205 & 1234 \\
Krankenhäuser und Pflege & 445 & 460 & 465 & 467 & 471 \\
Sparkassen & 220 & 213 & 213 & 209 & 203 \\
Ver- und Entsorgung & 155 & 160 & 160 & 160 & 158 \\
Nahverkehr & $100^{\text {a }}$ & 72 & 73,8 & 75 & 75 \\
Flughäfen & & 24 & 24 & 24 & 24 \\
Gesamt & $\mathbf{2 1 2 0}$ & $\mathbf{2 1 0 0}$ & $\mathbf{2 1 2 5 , 8}$ & $\mathbf{2 1 4 0}$ & $\mathbf{2 1 6 5}$ \\
\hline
\end{tabular}

a 2010 einschließlich Flughäfen; Quellen: VKA (2012a, 2014b, 2015c, 2016, 2017)

Die von der VKA publizierten Daten zum Personalbestand, der Tarifbeschäftigte und Auszubildende kommunaler Arbeitgeber in Verbandstarifbindung umfasst, zeigen kaum Veränderungen in den letzten drei Jahren. Seit 2007, als der Personalbestand mit 1,976 Mio. Beschäftigten seinen Tiefststand erreichte, stiegen die Beschäftigtenzahlen leicht, aber stetig wieder an (VKA, 2013, S. 3), bis 2016 mit 2,165 Mio. der höchste Personalbestand der vergangenen zehn Jahre erreicht wurde (VKA, 2017). ${ }^{2}$

Da wesentliche Strukturveränderungen im öffentlichen Sektor bereits in den 1990er Jahren stattfanden, ist es sinnvoll, die Beschäftigtenentwicklung im Langzeittrend zu beobachten. Auf Basis von Daten des Statistischen Bundesamtes zeigt sich zwischen 1992 und 2010 ein massiver Personalabbau (Keller, 2014a, S. 34), der seine Gründe zum einen in der Verschlankung des Verwaltungsapparats und der Zusammenlegung von Gebietskörperschaften in den neuen Bundesländern (Rosdücher, 1994) und zum anderen in der (Teil-)Privatisierung ehemals öffentlicher Dienstleistungen hat. Der Personalrückgang war bei den Kommunen deutlich stärker als bei Bund und Ländern. Gleichzeitig stieg die Beschäftigung im mittelbaren öffentlichen Dienst, der neben den Sozialversicherungsträgern, der Bundesbank und der Agentur für Arbeit weitere Einrichtungen in öffentlich-rechtlicher Trägerschaft einschließt, deutlich an. Neben dem Wegfall öffentlicher Beschäftigung spricht dies zum Teil auch für eine Beschäftigungsverlagerung vom kommunalen in den mittelbaren öffentlichen Dienst.

\subsection{Innerverbandliche Interessenbearbeitung und Spartenorientierung}

Zur innerverbandlichen Interessenbearbeitung werden die Mitglieder verschiedenen Gruppen, den Sparten, zugeordnet. Die VKA bildet für die einzelnen Sparten Gruppenausschüsse, die weitgehend autonom in der Vorbereitung tarifpolitischer Entscheidungen für die jeweils eigenen Mitglieder sind. Die Gruppenausschüsse der VKA konstituieren sich aus

2 Um die Auswirkungen der Privatisierungsbewegungen auf die Spartenstruktur der VKA demonstrieren zu können, wären diese Strukturdaten in einer Langzeitperspektive seit Anfang/Mitte der 1990er Jahre hilfreich. Leider liegen diese Informationen für frühere Jahrgänge nicht vor bzw. sind nicht zugänglich. 
nach differenziertem Stimmrecht gewählten Vertretern aus den entsprechenden Verbandsgruppen der regionalen KAV (Keller, 2010, S. 46) und erfüllen wichtige „Zulieferer- und Vorbereitungsfunktionen“ (ebd.) für die Verbandspolitik, indem sie die besonderen Problemlagen in den Mitgliedergruppen aufgreifen, sie in die Gremien des Dachverbandes transportieren und dadurch den innerverbandlichen Informationsfluss sicherstellen.

Derzeit gibt es auf Dachverbandsebene Gruppenausschüsse für Verwaltungen, für Krankenhäuser und Pflegeeinrichtungen, für Sparkassen, für Ver- und Entsorgungsbetriebe, für Nahverkehrsunternehmen und für Flughäfen. Zum Teil sind diese weiter in Unterausschüsse ausdifferenziert. In den regionalen KAV kann der Gruppenzuschnitt entsprechend der jeweiligen Mitgliederstruktur variieren. Zudem wirkt sich dort die Größe auf die Gruppenbildung aus. Während mitgliederstarke KAV die Interessenbearbeitung relativ stark gruppenspezifisch organisieren, sinkt mit abnehmender Verbandsgröße der Differenzierungsbedarf, da, wie aus einem kleinen KAV berichtet wird, die direkten Kommunikationswege zu den Mitgliedern ausreichen und eine Gruppenbildung nicht in der Satzung verankert werden müsse.

„Also wir haben das nicht gemacht. Unser früherer Geschäftsführer hat immer zu Recht gesagt: ,Dafür sind wir zu klein“." (Interview Geschäftsführer (GF) KAV klein)

Die regionalen KAV unterscheiden sich zudem in ihrer Mitgliederzusammensetzung. Während in einigen das Spektrum breit ist und damit die Kommunalverwaltungen weniger dominant sind, stellen diese in anderen die mit Abstand größte Mitgliedergruppe. Neben regionalen Besonderheiten (etwa hinsichtlich des Vorkommens an Häfen/Flughäfen) führt auch das Ausmaß an Privatisierung und Outsourcing zu unterschiedlichen Mitgliederstrukturen. In einigen KAV befinden sich die im Wettbewerb stehenden Mitglieder noch relativ stark in der Verbands- und Tarifbindung (was in den KAV als Erfolg der Verbandstätigkeit gilt), während in anderen große Teile der Dienstleistungsversorgung offenbar nicht mehr durch KAV-Mitglieder erfolgen. Daher sind dort die Anteile der im Wettbewerb stehenden Mitgliedergruppen eher klein (vgl. Abbildung 1). Nach der Schilderung eines Befragten ist die Dominanz der Kommunalverwaltungen in den ostdeutschen KAV in der Tendenz stärker als in den westdeutschen.

Die Mitgliederstruktur hat auch Konsequenzen für die innerverbandlichen Willensbildungsprozesse. Kommunale Arbeitgeberverbände, in denen die Verwaltungen dominieren, unterscheiden sich von jenen mit hohen Anteilen im Wettbewerb agierender Mitglieder, da der Bedarf an in Wettbewerb hilfreichen Tarifregelungen unterschiedlich verteilt ist.

„Also es nützt ja auch nichts, wenn man sagt: ,Wir brauchen wettbewerbsorientierte Regelungen' und Sie haben einen KAV, der, sagen wir mal 80 oder 90 Prozent Verwaltungen hat. Da mag es sein, dass da einfach in der Ausrichtung, in der Intention, eine andere Schwerpunktbildung ist.“ (Interview GF KAV groß)

Diese Aussage eines KAV-Geschäftsführers deutet darauf hin, dass aus der unterschiedlichen Mitgliederzusammensetzung Interessenkonflikte bei den verbandlichen Willensbildungsprozessen auf Dachverbandsebene resultieren können. 
Abbildung 1: Mitgliederstruktur KAV Brandenburg und KAV NW (Angaben in Prozent)



Quellen: KAV NW, 2016, S. 85; http://www.kav-brandenburg.de/?page_id=3628 (geprüft am 09.03. 2017)

Die autonome Interessenbearbeitung der einzelnen Mitgliedergruppen, die Spartenorientierung, setzt sich auch in den Tarifverträgen der VKA fort. Mit dem Abschluss des TVöD im Jahr 2005 entfiel die Unterscheidung von Arbeitern und Angestellten (Schmidt et al., 2011, S. 47ff). Tarif- und arbeitsrechtliche Fragen werden seitdem zunehmend innerhalb der Sparten bearbeitet. Dies äußert sich in den gesonderten Fassungen des TVöD, in denen der Flächentarifvertrag in den besonderen Teilen auf die Bedarfe der Mitgliedersegmente zugeschnitten wird. Zudem existieren mit dem Tarifvertrag Versorgungsbetriebe (TV-V) und dem mit dem Marburger Bund vereinbarten TV Ärzte/VKA zwei weitere bundesweit gültige Spartentarifverträge.

Eine gewisse Spartenorientierung hat nach Aussage eines VKA-Vertreters Tradition, Sparkassen und Krankenhäuser hätten sich seit jeher von den Verwaltungen unterschieden. Mit der Modernisierung des öffentlichen Dienstes wuchs aber die Bedeutung der Sparten, als, ausgelöst durch die Liberalisierung im Energiesektor, die Stadtwerke in den Wettbewerb mit privaten Anbietern gerieten.

„Als wir in den 1990er Jahren bezogen auf die Stadtwerke mit dem TV-V begonnen haben, da ist es zum erstem Mal, dieses Thema Spartenorientierung, so ganz klassisch und bewusst genannt worden. [...] Das hat aber auch mit der Veränderung des Umfeldes insgesamt zu tun.“ (Interview GF VKA)

Zudem kam es nach der Tarifreform zu einem Bedeutungsaufschwung der regionalen KAV bei der Umsetzung von Tarifverträgen. Tarifliche Öffnungsklauseln, die landesbezirklich zu konkretisieren sind, wurden in den Flächentarif integriert und führten zu einer Dezentralisierung der Umsetzungsverantwortung. In der Nahverkehrssparte werden die Verhandlungen zum Tarifvertrag für die kommunalen Nahverkehrsunternehmen (TV-N) rein landesbe- 
zirklich geführt. Die VKA greift dort nur bei Fragen von übergeordnetem Interesse ein, so etwa beim Abschluss des seit 2013 in fünf Bundesländern geltenden Tarifvertrags zur Gestaltung des demografischen Wandels bei den kommunalen Nahverkehrsunternehmen, dem TV Demografie Nahverkehr.

\section{Struktureller Wandel im öffentlichen Sektor und die Rolle kommunaler Arbeitgeberverbände}

\subsection{Liberalisierung, Privatisierung, Outsourcing, Rekommunalisierung}

Für die Arbeitsbeziehungen im öffentlichen Sektor wurde in einer Reihe von Studien gezeigt (Brandt \& Schulten, 2008; Hermann \& Flecker, 2012), dass die Privatisierung vor allem bei den Nachfolgern der ehemaligen Staatsmonopole Post und Bahn, aber auch in den Wettbewerbssparten der VKA eine Spaltung der Beschäftigungsbedingungen bewirkte, indem sich zwei Konfliktlinien auftaten. Auf der Branchenebene verblieben bei den Monopolnachfolgern (Deutsche Bahn, Deutsche Post, Telekom) weitgehend zentralisierte Arbeitsbeziehungen und vergleichsweise hohe Tarifstandards, während sich bei den neuen Wettbewerbern oft dezentralisierte, fragmentierte, häufig aber auch gar keine institutionalisierten Arbeitsbeziehungen mit im Ergebnis schlechteren Beschäftigungskonditionen entwickelten. Auf der betrieblichen Ebene eröffnet sich dadurch eine zweite Konfliktlinie, die regelmäßig die zu schlechteren Konditionen neueingestellten Beschäftigten zu Modernisierungsverlierern macht, wogegen die Altbeschäftigten meist durch Besitzstandsregelungen geschützt sind.

Für die Kommunalverwaltungen brachte die Einführung von TVöD bzw. TV-L zwar ebenfalls eine gewisse Besitzstandssicherung für bereits zuvor tätige Beschäftigte, doch derartige dezentralisierungsbedingte Ausfransungserscheinungen in den Arbeitsbeziehungen zeichnen sich bislang nicht ab. Zwar gibt es durch die seit 2005 unterschiedliche Tarifentwicklung bei Bund/Kommunen und Ländern ebenfalls Anzeichen einer Dezentralisierung (Keller, 2014a; 2010), jedoch in einer „kontrollierten“ Variante (Traxler, 1995) und ohne das Entstehen tariffreier Zustände.

Festgestellt wurde aber auch, dass sich die erhofften Privatisierungsvorteile nicht im gewünschten Ausmaß einstellten. Seit einigen Jahren sind gegenläufig Rekommunalisierungstendenzen erkennbar (Libbe, Hanke \& Verbücheln, 2011), da viele Kommunen erkannten, dass kommunale Dienstleistungserbringung vorteilhaft sein kann (Richter, 2013). Die Position kommunaler Arbeitgeberverbände zur Rekommunalisierung ist jedoch nicht ganz ohne Vorbehalt. Die Rückführung öffentlicher Dienste in kommunale Verantwortung wird befürwortet, da jedoch ein ordnungspolitisches Interesse an der Mitgliederrekrutierung und der Organisierung von Tarifbindung besteht, wird Rekommunalisierung nur dann umstandslos begrüßt, wenn daraus auch die Verbandsmitgliedschaft folgt.

„Das Thema Rekommunalisierung, da bin ich immer etwas sensibel, weil das Thema Rekommunalisierung beantwortet in dem Kontext noch nicht jede Frage. Denn: Wenn jemand rekommunalisiert, ist damit noch nicht beantwortet, wie er rekommunalisiert, ob er in unser Tarifrecht rekommunalisiert oder auch nicht." (Interview GF VKA) 


\subsection{Neue Verhandlungspartner}

Zwar nicht als Ursache, jedoch als Begleiterscheinung von Liberalisierung und Privatisierung wird auch die Ausdifferenzierung der Gewerkschaftslandschaft diskutiert. Neben dem traditionell im öffentlichen Dienst existierenden „Koalitionspluralismus“ (Keller, 2014b, S. 316) traten die Spartengewerkschaften berufsständischen Ursprungs (Schroeder, Kallas \& Greef, 2011) als neue Verhandlungspartner auf. Verbandsvertreter unterscheiden in den sozialpartnerschaftlichen Beziehungen zwischen den traditionellen Gewerkschaften des öffentlichen Dienstes (ver.di, früher ÖTV) sowie dem Tarifflügel des Beamtenbundes (dbb tarifunion) und den aufstrebenden Spartengewerkschaften Marburger Bund, Gewerkschaft der Lokführer GdL und Gewerkschaft der Flugsicherung GdF. Von den genannten Gewerkschaften kam der Marburger Bund als direkter Verhandlungspartner der VKA hinzu. Die GdL ist unter dem Dach der dbb tarifunion organisiert, tritt jedoch auch als Einzelgewerkschaft als Verhandlungspartner einiger regionaler KAV in Erscheinung (z.B. im Saarland). Die GdF hingegen ist eine 2004 neu gegründete Gewerkschaft, die als Tarifpartei die Interessen der Beschäftigten der Flugsicherung gegenüber einzelnen KAV-Mitgliedern, wie der Fraport AG, vertritt. Die Beziehungen mit den letztgenannten Organisationen werden (noch) als schwierig beschrieben, da sie - interessenpolitisch pointiert - nach Darstellung eines KAV-Geschäftsführers eine höhere Konflikthaftigkeit aufwiesen, die weniger dem Interesse am Verhandlungsergebnis als vielmehr dem Motiv der Mitgliederrekrutierung folge.

„Eine Gewerkschaft, die Zoff macht, die bekommt Mitglieder.“ (Interview GF KAV groß)

Für die Geschäftsstellen bedeutet das Aufkommen neuer Verhandlungspartner einen erhöhten Verhandlungsaufwand und damit auch ein Mehraufkommen an Vor- und Nachbereitungsarbeit. Als schwerwiegender bewerten die interviewten Verbandsvertreter jedoch die Folgen für die Mitglieder, da die mit Tarifverträgen verbundene Friedenspflicht leiden würde, wenn man sich fortlaufend in Verhandlungen befände.

Wegen verfassungsrechtlicher Bedenken hinsichtlich eines zu starken Eingriffs in die Koalitionsfreiheit (Däubler, 2015) wurden von kleineren Gewerkschaften und auch der dbb tarifunion Klagen gegen das Tarifeinheitsgesetz beim Bundesverfassungsgericht eingereicht, denen die Rechtsprechung letztlich nicht gefolgt ist.

\section{Strategische Bearbeitung des Strukturwandels}

\subsection{Flexibilisierung der Mitgliedschaftsformen}

Arbeitgeberverbänden stehen als Instrument der Mitgliedschaftslogik verschiedene Möglichkeiten der Bearbeitung strukturellen Wandels offen. Wie eingangs erläutert, setzen sie zur Stabilisierung der Organisationsstärke und zur Vermeidung von „Verbandsflucht“ seit den 1990er Jahren auf flexible Mitgliedschaftsformen. Im Organisationsbereich der VKA gibt es bislang keine im Ausmaß mit der Privatwirtschaft vergleichbare Entwicklung. OTVerbände existieren nicht, Mitgliedschaften ohne Tarifbindung sind die Ausnahme. Mit einem traditionell hohen Organisationsgrad zwischen 80 und 90 Prozent (Keller, 2010, S. 59) existieren auch die Organisationsprobleme der Privatwirtschaft nicht in vergleichbarer Wei- 
se, zumal sich auch die nicht organisierten Arbeitgeber in hohem Maße an den Verbandstarifen orientieren. Zwar sehen auch die KAV Gastmitgliedschaften vor, wobei es jedoch zwischen den Regionalverbänden Unterschiede hinsichtlich der Wahlfreiheit zwischen ordentlicher und OT-Mitgliedschaft gibt. Die Kriterien, nach denen Gast- oder OTMitgliedschaften ermöglicht werden, sind dabei unterschiedlich restriktiv.

„Es gibt kommunale Arbeitgeberverbände, die lassen nach wie vor keine OT-Mitglieder zu. Es gibt kommunale Arbeitgeberverbände, die lassen OT-Mitgliedschaften zu, auch wenn originäre Mitgliedschaft möglich wäre, also Tarifgebundenheit möglich wäre. Und es gibt welche, die sagen: ,Wir lassen OT-Mitgliedschaft zu, aber nur da, wo es keine Tarifmöglichkeit gibt, wo keine Tarifbindung herstellbar ist.' Und deshalb ist die Entwicklung bei den Gastmitgliedern unterschiedlich, zum Teil gibt es da durchaus auch Zuwächse." (Interview GF VKA)

Primär wird das Interesse artikuliert, über die Vollmitgliedschaft „Tarifbindung“ zu organisieren. Allerdings wird die Eröffnung der OT-Mitgliedschaftsoption durch die regionalen KAV vom Dachverband nicht durch entsprechende Satzungsvorschriften verhindert. Dass diese Offerte in den Wettbewerbssparten auch genutzt wird, zeigt sich z.B. beim KAV Brandenburg, bei dem in der Sparte der Krankenhäuser und Pflegeeinrichtungen lediglich eines der 29 Mitglieder tarifgebunden ist (KAV Brandenburg, 2017). Das Beispiel demonstriert, dass auch kommunale Arbeitgeberverbände im Grundsatz über das Instrumentarium flexibilisierte Mitgliedschaftsformen zur Lösung von Organisationsproblemen verfügen.

Die eingangs formulierte Erwartung größerer Stabilität in der Verbandsstruktur findet offenbar nur noch bei der größten Sparte, den Kommunalverwaltungen, weitgehend Bestätigung. Der freiwillige Tarifverzicht als Strategie der Mitgliedschaftslogik ist bei kommunalen Arbeitgeberverbänden somit durchaus erkennbar, aber derzeit noch weniger stark ausgeprägt. Allerdings gibt es in der Binnenstruktur auch bei diesen Verbänden Entwicklungen, die sich als Annäherung an das privatwirtschaftliche Modell interpretieren lassen. Neben der moderaten Ausbreitung tariffreier Mitgliedschaften in den Wettbewerbssparten kommt ein zweiter Aspekt der strukturellen Anpassung zum Tragen. Die KAV nehmen zwar keine privaten Arbeitgeber in die Mitgliedschaft auf und weiten folglich ihre Organisationsdomäne nicht aus, haben sich aber auf die Folgen von Privatisierung und Outsourcing insofern eingestellt, als sich der private Einfluss durch die Anreicherung der gemischtwirtschaftlichen Unternehmen mit privatem Kapital erhöht hat. Somit deutet sich ein binnenstruktureller Prozess der „Verprivatwirtschaftlichung“ zumindest der im Wettbewerb stehenden Sparten an.

Für die kommunalen Arbeitgeberverbände kann aus den beschriebenen Tendenzen durch eine schärfere Abgrenzung der Wettbewerbssparten von den Verwaltungen eine Diversifizierung der Mitgliederinteressen resultieren, die zu einer Verfestigung der Spartenorientierung führen kann. Für die Verbände selbst wäre damit ein erhöhter Kommunikations- und Abstimmungsaufwand bei der Vereinheitlichung der Mitgliederinteressen ebenso verbunden wie eine Steigerung der Anforderungen bei der Verpflichtung auf eine gemeinsame Verbandspolitik.

\subsection{Verbandspolitischer Output}

Die Hauptaufgabe kommunaler Arbeitgeberverbände besteht im Verhandeln und im Abschluss von Tarifverträgen. Wenn die kommunalwirtschaftlichen Sparten durch Liberalisie- 
rung und Privatisierung in starken Konkurrenzdruck mit privaten Anbietern geraten bzw. durch politisch vorgelagerte Entscheidungen in ihn manövriert werden, dann werden die Lohnkosten zu einem relevanten Wettbewerbsfaktor. Daher ist es für kommunale Arbeitgeberverbände im Olson'schen Sinne rational, den Verbandsoutput an die veränderten Rahmenbedingungen anzupassen und über konkurrenzfähige Tarifverträge Mitgliedschaftsanreize zu setzen.

Dies erfolgt durch tarifliche Öffnungsklauseln, wie sie es etwa in den Eingruppierungsbestimmungen des TVöD gibt. Nach § 15 Abs. 3 TVöD können auf der Basis landesbezirklicher Tarifvereinbarungen ,für an- und ungelernte Tätigkeiten in von Outsourcing und/oder Privatisierung besonders bedrohten Bereichen in den Entgeltgruppen 1 bis 4 Abweichungen von der Entgelttabelle bis zu einer dort vereinbarten Untergrenze vorgenommen werden.“ Zur tatsächlichen Nutzungshäufigkeit dieses von Verbandsvertretern als „Knautschzone“ bezeichneten Instruments liegen leider keine belastbaren Informationen vor. Dass es in der Intention kommunaler Arbeitgeberverbände liegt, mit der Bereitstellung wettbewerbsfähiger Tarifverträge Konkurrenzfähigkeit zu ermöglichen und dadurch Anreize zum Verbleib im Verband zu setzen, wird jedoch von hochrangigen Verbandsvertretern unterstrichen.

„Man muss ein Produkt anbieten, das auch angenommen wird. Und das funktioniert nur, wenn mit diesem
Produkt auch Wettbewerbsfähigkeit organisiert werden kann. Und viele Dinge im TVöD hängen ja genau
damit zusammen, dieser $\S 15$ Absatz 3 TVöD. [...] Und das ist, glaube ich, entscheidend, Angebote, Tarif-
verträge anzubieten, die öffentliche Aufgabenwahrnehmung zukunftsorientiert weiter ermöglichen.“" (Inter-
view GF VKA)

Darüber hinaus beinhaltet der TVöD mit dem $\S 18$ Regelungen zur leistungsorientierten Bezahlung, die, dem Modernisierungsdiskurs des Neuen Steuerungsmodells folgend, als eine der zentralen Arbeitgeberforderungen bei der Tarifreform umgesetzt wurden (Schmidt \& Müller, 2013). Damit sollte der Erwartung effektiverer und effizienterer Aufgabenerledigung Rechnung getragen werden.

Zudem nutzen kommunale Arbeitgeberverbände in Reaktion auf die Wettbewerbskonstellation auf Facharbeitsmärkten einseitige Arbeitgeberrichtlinien, von denen derzeit zwei gelten. Seit April 2012 ist die Richtlinie zur Gewinnung und Bindung der Ärzte im öffentlichen Gesundheitsdienst (Fachärzte-ÖGD-Richtlinie) in Kraft, die auf den - bislang ungelösten - Konflikt zwischen der VKA und dem Marburger Bund hinsichtlich der tarifvertraglichen Einordnung der Ärzte im öffentlichen Gesundheitsdienst zurückgeht (VKA, 2012b, S. 7f). Sie erlaubt einzelfallbezogene Zulagen zur Verhinderung der Abwanderung von Fachkräften. Außerdem ist aktuell die 2011 erlassene Richtlinie zur Gewinnung und Bindung von Fachkräften auf dem Gebiet der Informationstechnik (IT-Richtlinie) gültig, deren Erlass mit veralteten Eingruppierungsbestimmungen begründet wird. Sie diente damit der Kompensation einer tariflichen Regelungslücke in der bis Ende 2016 gültigen Entgeltordnung. Daher mag die erneute Verlängerung der IT-Richtlinie in der VKA-Mitgliederversammlung vom 16. November 2016 verwundern, da die Eingruppierungsbestimmungen bereits mit der Verständigung auf eine Entgeltordnung in der Tarifeinigung der Kommunen vom 29. April 2016 neu geregelt wurden. Diese traten zum 1. Januar 2017 in Kraft. Die Verlängerung der IT-Richtlinie spricht somit für weiterhin bestehende Rekrutierungs- und Bindungsprobleme bei IT-Fachkräften, die für kommunale Arbeitgeber offenbar allein mit den bestehenden tariflichen Entgeltbestandteilen nicht zu lösen sind. 
Arbeitgeberrichtlinien ermöglichen die kurzfristige Reaktion auf Personalengpässe und sind nur ,in begründeten Einzelfällen“ (VKA, 2015a; VKA, 2015b) anzuwenden. Dies liegt an der Besonderheit im öffentlichen Dienst, dass tariflich vereinbarte Mindestnormen zugleich Höchstnormen sind. ${ }^{3}$ Kommunale Arbeitgeber, die übertarifliche Entgelte zahlen, verhalten sich nicht regelkonform und müssten mit Verbandsstrafen rechnen. Die Arbeitgeberrichtlinien haben somit auch die Funktion, satzungswidrige betriebliche Entgeltpraktiken zu legitimieren.

Mit Blick auf die Mitgliedschaftslogik kommunaler Arbeitgeberverbände offenbart die Analyse der Verbandsprodukte, dass tarifliche Regelungen meist angepasst bzw. weiterentwickelt werden, wenn sich die Rahmenbedingungen bei den Mitgliedern verändern, etwa durch verstärkten Wettbewerb infolge privatisierter Dienstleistungserbringung. Das Beispiel der Arbeitgeberrichtlinien zeigt aber, dass kommunale Arbeitgeberverbände zu innovativen Problemlösungen fähig sind, indem sie bei besonderen Problemlagen oder beim Fehlen von Tarifnormen aktiv steuern und damit Handlungsfähigkeit gewährleisten können. Damit bewirken sie auch eine Stärkung ihrer Organisationsfähigkeit, indem sie durch die Bereitstellung problemadäquater Rahmenbedingungen die Anreizfunktion zur Verbandsmitgliedschaft beleben.

\section{Fazit}

Einleitend wurde die Frage gestellt, in welcher Weise sich die infolge der Liberalisierung von Produkt- und Dienstleistungsmärkten aufgetretene (Teil-)Privatisierung öffentlicher Dienste im Verbandshandeln kommunaler Arbeitgeberverbände niederschlägt. Bei der Analyse wurde deutlich, dass diese auch in die öffentliche Aufgabenwahrnehmung eine enorme Dynamik brachte. Umbrüche im (binnen-)organisatorischen Setting kommunaler Arbeitgeberverbände waren die Folge, indem die Mitgliedersparten Autonomiegewinne zulasten der Gesamtorganisation beanspruchen konnten. Damit ging auch ein Bedeutungszuwachs der regionalen KAV einher, die in vielen Fragen Zuständigkeit erlangten, die früher bundeseinheitlich geregelt wurden. Der Bundesangestelltentarifvertrag (BAT) sowie die Rahmentarifverträge für Gemeindearbeiter (BMT-G) gaben über Jahrzehnte hinweg die Beschäftigungsbedingungen im kommunalen öffentlichen Dienst vor, ließen sich quasi-automatisch umsetzen und boten kaum Anlass für tiefgreifende Änderungen. Erst in den 1990er Jahren, auch beflügelt von der Wiedervereinigung und deren Folgen für das öffentliche Beschäftigungssystem, kam Bewegung in die Arbeitsbeziehungen des öffentlichen Sektors, indem auch sie im Zuge der allgemeinen Kritik des öffentlichen Dienstes einem Veränderungsdiktat unterworfen wurden. Einen Höhepunkt fand diese Entwicklung schließlich im Abschluss des TVöD als tarifrechtliche Reaktion der Sozialpartner auf das Modernisierungserfordernis im öffentlichen Dienst.

Fortan gezwungen, sowohl die Verbandsstruktur als auch die tarifpolitische Ausrichtung auf die veränderten Rahmenbedingungen hin zu überprüfen, zeigt sich, dass kommunale Arbeitgeberverbände verstärkt mit dem Konkurrenzdruck und Wettbewerb ihrer Mitglieder

3 Laut Satzung der VKA muss jeder Mitgliedsverband seine Mitglieder durch Satzungsvorschriften dazu verpflichten, „die von der VKA abgeschlossenen Tarifverträge durchzuführen und nicht zu überschreiten“ (VKA, 2014a, S. 5). 
umgehen müssen. Anpassungsreaktionen finden als Ausdruck der Mitgliedschaftslogik zum einen in der Binnenstruktur statt, indem die spartenbezogene Problembearbeitung zugenommen hat und der privatwirtschaftliche Einfluss durch die Anreicherung kommunaler Dienstleistungserbringung mit privatem Kapital zugelassen wurde. Zum anderen gibt es Anpassungsreaktionen dahingehend, dass die veränderten Wettbewerbsbedingungen auch in den „Produkten“ adaptiert werden. Mit Abschluss des TVöD und in den Spartentarifverträgen der Versorgungsbetriebe, der Nahverkehrsunternehmen und der Krankenhäuser finden sich verstärkt Öffnungsklauseln als Flexibilisierungselemente, die Wettbewerbsfähigkeit sichern sollen. Hinzu kommen Arbeitgeberrichtlinien, die Konkurrenzfähigkeit auf Teilarbeitsmärkten bei der Fachkräfterekrutierung und -bindung ermöglichen sollen.

Als Interessenorganisation handeln die kommunalen Arbeitgeberverbände vornehmlich im Mitgliederinteresse. Es ist eine gewisse Nachfrageorientierung sowohl im Verbandsaufbau als auch im Verbandsoutput zu konstatieren. So folgen die innerverbandliche Arbeitsteilung und die Strukturierung der Mitgliedersegmente auch nicht der Initiative der Verbandsspitze, sondern dem Mitgliederinteresse. Veränderungen in der Spartenaufteilung oder die Bildung von Unterausschüssen innerhalb einzelner Sparten erfolgen in der Regel dann, wenn sich durch gesetzliche, tarifrechtliche oder sonstige Gründe spezifische Problemlagen für spezielle Mitglieder stellen oder verändern. Durch die strukturelle Mitgliederdifferenzierung gewährleisten kommunale Arbeitgeberverbände eine auf die Mitgliederbedarfe zugeschnittene Organisationsform bei der Problembearbeitung. Dazu gehört, dass es im Vergleich zur Privatwirtschaft bislang keine übermäßige Ausbreitung tariffreier Mitgliedschaften gab. Die Analyse zeigt, dass die Initiative zur Veränderung meist nicht von den kommunalen Arbeitgeberverbänden selbst ausgeht. Entsprechend ihrer Rolle im Institutionengefüge des öffentlichen Dienstes sind sie weitgehend auf ihre Funktion als Tarifvertragspartei reduziert.

\section{Literatur}

Bahnmüller, R. \& Hoppe, M. (2014). Weiterbildung in Kommunalverwaltungen. Bestandsaufnahme, tarifliche Regelungen, Empfehlungen. Reihe Modernisierung des öffentlichen Sektors. Sonderband 44. Berlin: edition sigma. doi:10.5771/9783845269856

Behrens, M. (2013). Arbeitgeberverbände. In H. Hirsch-Kreinsen, H. Minssen (Hrsg.), Lexikon der Arbeits- und Industriesoziologie (S. 59-64). Berlin: edition sigma.

Behrens, M. (2011). Das Paradox der Arbeitgeberverbände. Von der Schwierigkeit, durchsetzungsstarke Unternehmensinteressen kollektiv zu vertreten. Berlin: edition sigma. doi: $10.5771 / 9783845269559$

Bogumil, J. (2014). 20 Jahre Neues Steuerungsmodell - Eine Bilanz. In E. Wiechmann \& J. Bogumil (Hrsg.), Arbeitsbeziehungen und Demokratie im Wandel. Festschrift für Leo Kißler (S. 41-58). Baden-Baden: Nomos.

Bosch, G. (2013). Public sector adjustments in Germany: From cooperative to competitive federalism. In D. Vaughn-Whitehead (Hrsg.), Public Sector Shock (S. 214-258). Cheltenham u.a.: Edward Elgar Publishing Ltd.

Bosch, G., Mesaros, L., Schilling G. \& Weinkopf, C. (2012). The public sector pay system and public procurement in Germany. National report. University of Duisburg. IAQ.

Brandt, T. \& Schulten, T. (2008). Liberalisierung und Privatisierung öffentlicher Dienstleistungen und die Erosion des Flächentarifs. WSI-Mitteilungen, 61 (10), 570-576. 
Czerwick, E. (2008). Reformen des öffentlichen Dienstrechts in Deutschland seit 1997: Ansätze und Schwerpunkte. In W. H. Loring (Hrsg.), Moderne Verwaltung in der Bürgergesellschaft (S. 6684). Baden-Baden: Nomos. doi:10.5771/9783845209630-66

Däubler, W. (2015). Gutachten zum Gesetzesentwurf der Bundesregierung zum Tarifeinheitsgesetz. Erstellt im Auftrag der Bundestagsfraktion DIE LINKE. Berlin.

Engartner, T. (2008). Privatisierung und Liberalisierung - Strategien zur Selbstentmachtung des öffentlichen Sektors. In C. Butterwegge, B. Lösch \& R. Ptak (Hrsg.), Kritik des Neoliberalismus. 2., verbesserte Auflage (S. 87-133), Wiesbaden: VS Verlag. doi:10.1007/978-3-531-90932-5_3

Gläser, J. \& Laudel, G. (2010). Experteninterviews und qualitative Inhaltsanalyse. 4. Auflage. Wiesbaden: VS Verlag. doi:10.1007/978-3-531-91538-8

Gottschall, K., Kittel, B., Briken, K., Heuer, J.-O., Hils, S., Streb, S. \& Tepe, M. (2015). Public Sector Employment Regimes - Transformations of the State as an Employer. Basingstoke, New York: Palgrave. doi:10.1057/9781137313119

Haipeter, T. (2017). OT-Mitgliedschaften und OT-Verbände. In W. Schroeder \& B. Weßels (Hrsg.), Handbuch Arbeitgeber- und Wirtschaftsverbände in Deutschland. 2. Aufl. (S. 305-317). Wiesbaden: VS Verlag. doi:10.1007/978-3-658-08176-8_12

Hebeler, T. (2008). Verwaltungspersonal. Eine rechts- und verwaltungswissenschaftliche Strukturierung. Baden-Baden: Nomos. doi:10.5771/9783845205731

Hebeler, T., Sitzer, A. \& Tews, J. (2012). Ökonomisierung des öffentlichen Dienstrechts - eine Bestandsaufnahme. Die Personalvertretung, 55 (2), 44-50.

Henneberger, F. (2017). Zentrale Theorien und etwas Empirie zur Analyse des kollektiven Handelns von Unternehmern. In W. Schroeder \& B. Weßels (Hrsg.), Handbuch Arbeitgeber- und Wirtschaftsverbände in Deutschland. 2. Aufl. (S. 173-198), Wiesbaden: VS Verlag. doi:10.1007/978-3-658-08176-8_7

Hermann, C. \& Flecker, J. (Hrsg.). (2012). Privatization of Public Services. Impacts for Employment, Working Conditions, and Service Quality in Europe. New York: Routledge.

Hirschman, A. O. (1974). Abwanderung und Widerspruch. Tübingen: J.C.B. Mohr (Paul Siebeck).

Hyman, R. (2008). The State in Industrial Relations. In P. Blyton, N. Bacon, J. Fiorito \& E. Heery (Hrsg.), The SAGE Handbook of Industrial Relations (S. 258-284), Los Angeles, London, New Delhi, Singapore: SAGE. doi:10.4135/9781849200431.n14

KAV Brandenburg (2017). Kranken- und Pflegeeinrichtungen. Abgerufen von https://www.kav-brandenburg.de/?page_id=373 (geprüft am 09.03.2017).

KAV NW (2016). Jahresbericht 2015/2016. Wuppertal.

Keller, B. (2010). Arbeitspolitik im öffentlichen Dienst. Reihe Modernisierung des öffentlichen Sektors, Sonderband 36. Berlin: edition sigma. doi: 10.5771/9783845269771

Keller, B. (2013). Die Arbeitgeber des öffentlichen Sektors: Institutionelle Stabilität und sozioökonomischer Wandel. WSI-Mitteilungen, 66, 500-509.

Keller, B. (2014a). Der öffentliche Dienst in der Finanz- und Schuldenkrise, Austeritätspolitik und die Entwicklung der Arbeitsbeziehungen. Sozialer Fortschritt, 63 (1-2), 30-39. doi:10.3790/sfo.63.1-2.30

Keller, B. (2014b). Gewerkschaften und Interessenverbände im System der Arbeitsbeziehungen des öffentlichen Dienstes. In W. Schroeder (Hrsg.), Handbuch Gewerkschaften in Deutschland. 2. Aufl. (S. 311-335), Wiesbaden: Springer VS.

doi:10.1007/978-3-531-19496-7_13

Keller, B. (2017). Arbeitgeberverbände des öffentlichen Sektors. In W. Schroeder \& B. Weßels (Hrsg.), Handbuch Arbeitgeber- und Wirtschaftsverbände in Deutschland, 2. Aufl. (S. 141-171), Wiesbaden: VS Verlag. doi:10.1007/978-3-658-08176-8_6 
Keller, B. \& Henneberger, F. (1992). Beschäftigung und Arbeitsbeziehungen im öffentlichen Dienst der neuen Bundesländer. Gewerkschaftliche Monatshefte, 43 (6), 331-342.

Keller, B., Due, J. \& Andersen, S. K. (2001). Employer Associations and Unions in the Public Sector. In C. Dell'Aringa, S. Bach \& B. Keller (Hrsg.), Strategic Choices in Reforming Public Service Employment (S. 71-96), Basingstoke, New York: Palgrave. doi:10.1057/9781403920171_4

KGSt (1993). Das Neue Steuerungsmodell: Begründung, Konturen, Umsetzung. KGSt Bericht 5/1993. Köln: KGSt.

Lamnek, S. (2010). Qualitative Sozialforschung, 5. Auflage. Weinheim: Beltz.

Libbe, J., Hanke, S. \& Verbücheln, M. (2011). Rekommunalisierung - Eine Bestandsaufnahme. Difu Papers. Berlin.

Mayring, P. (2015). Qualitative Inhaltsanalyse. 12., überarb. Auflage. Weinheim \& Basel: Beltz.

Mehde, V. (2011). Arbeitsbeziehungen im Rahmen der Modernisierung von Staat und Verwaltung. In R. Koch, P. Conrad \& W. H. Loring (Hrsg.), New Public Service. Öffentlicher Dienst als Motor der Staats- und Verwaltungsmodernisierung (2., überarbeitete und erweiterte Auflage, S. 129149). Wiesbaden: Gabler. doi:10.1007/978-3-8349-6371-0_7

Mühlenkamp, H. (2003). Zum grundlegenden Verständnis einer Ökonomisierung des öffentlichen Sektors - Die Sicht eines Ökonomen. In J. Harms \& C. Reichard (Hrsg.), Die Ökonomisierung des öffentlichen Sektors: Instrumente und Trends (S. 47-73), Baden Baden: Nomos.

Olson, M. (2004). Die Logik kollektiven Handelns (5. Auflage). Tübingen: Mohr Siebeck.

Rehder, B. (2009). Die neue Dominanz der Mitgliedschaftslogik - Interessenvermittlung in der Tarifpolitik. In B. Rehder, T. v. Winter \& U. Willems (Hrsg.), Interessenvermittlung in Politikfeldern. Vergleichende Befunde der Policy- und Verbändeforschung (S. 52-67), Wiesbaden: VS Verlag. doi:10.1007/978-3-531-91697-2_3

Rehm, H. \& Matern-Rehm, S. (2010). Kommunalfinanzen. Wiesbaden: VS Verlag.

Richter, P. (2013). Gefahr im „Vollzug“? Die Kommunalisierung staatlicher Aufgaben und ihre Auswirkung auf die Aufgabenerledigung. In M. Haus \& S. Kuhlmann (Hrsg.), Lokale Politik und Verwaltung im Zeichen der Krise? (S. 179-195). Wiesbaden: Springer VS. doi:10.1007/978-3-531-19160-7_10

Rosdücher, Jörg (1994). Kommunale Arbeitgeberverbände in den neuen Bundesländern. Zeitschrift für öffentliche und gemeinwirtschaftliche Unternehmen, 17 (4), 414-429.

Schmidt, W. \& Müller, A. (2013). Leistungsorientierte Bezahlung in den Kommunen. Befunde einer bundesweiten Untersuchung. Reihe Modernisierung des öffentlichen Sektors, Sonderband 42. Berlin: edition sigma. doi:10.5771/9783845269832

Schmidt, W., Müller, A. \& Trittel, N. (2011). Der Konflikt um die Tarifreform des öffentlichen Dienstes. Verhandlungsprozesse und Umsetzungspraxis. Reihe Modernisierung des öffentlichen Sektors, Sonderband 38. Berlin: edition sigma. doi:10.5771/9783845269795

Schmitter, P. C. \& Streeck, W. (1981). The Organization of Business Interests. A Research Design to Study the Associative Action of Business in the Advanced Industrial Societies of Western Europe. Berlin: WZB.

Schneider, K. \& Simon, N. (2014). Arbeiten im Interesse der Bürgerinnen und Bürger - Die Leistungsfähigkeit des öffentlichen Dienstes im Spannungsfeld von Bürger- und Beschäftigteninteressen. In J. Bogumil \& E. Wiechmann (Hrsg.), Arbeitsbeziehungen und Demokratie im Wandel (S. 84-97). Baden-Baden: Nomos.

Schroeder, W. (2007). Arbeitgeberverbände. In T. v. Winter \& U. Willems (Hrsg.), Interessenverbände in Deutschland (S. 197-220). Wiesbaden: VS Verlag. doi:10.1007/978-3-531-90602-7_7

Schroeder, W., Kallas, V. \& Greef, S. (2011). Berufsgewerkschaften in der Offensive. Vom Wandel des deutschen Gewerkschaftsmodells. Wiesbaden: Springer VS. doi:10.1007/978-3-531-92880-7

StatBA (2016). Personal des öffentlichen Dienstes. Fachserie 14, Reihe 6. Wiesbaden. 
Traxler, F. (1993). Gewerkschaften und Arbeitgeberverbände. Probleme der Verbandsbildung und Interessenvereinheitlichung. In W. Müller-Jentsch (Hrsg.), Konfliktpartnerschaft. Akteure und Institutionen der industriellen Beziehungen (2. Auflage, S. 141-167). München \& Mering: Hampp Verlag.

Traxler, F. (1995). Farewell to labour market associations? Organized versus disorganized decentralization as a map for industrial relations. In C. Crouch \& F. Traxler (Hrsg.), Organized Industrial Relations in Europe: What Future? (S. 3-19). Aldershot: Avebury.

VKA (2012a). Pressemappe der VKA zur Tarifrunde 2012. Tarifverhandlungen für die Beschäftigten von Bund und Kommunen. Frankfurt am Main.

VKA (2012b). VKA Nachrichten, 3/2012.

VKA (2013). VKA Nachrichten September 2013. Frankfurt am Main.

VKA (2014a). Satzung der Vereinigung der kommunalen Arbeitgeberverbände (VKA) vom 21. November 2008. Zuletzt geändert durch den Beschluss der Mitgliederversammlung vom 21. November 2014. Frankfurt am Main.

VKA (2014b). Jahresbericht 2014. Frankfurt am Main.

VKA (2015a). Arbeitgeberrichtlinie der VKA zur Gewinnung und zur Bindung der Fachärztinnen und Fachärzte im öffentlichen Gesundheitsdienst (Fachärzte-ÖGD-RL), in der Fassung des Beschlusses der Mitgliederversammlung der VKA vom 27. November 2015.

VKA (2015b). Arbeitgeberrichtlinie der VKA zur Gewinnung und zur Bindung von Fachkräften auf dem Gebiet der Informationstechnik (IT-RL), in der Fassung des Beschlusses der Mitgliederversammlung der VKA vom 27. November 2015.

VKA (2015c). Jahresbericht 2015. Frankfurt am Main.

VKA (2016). Jahresbericht 2016. Frankfurt am Main.

VKA (2017). Kurzinfo zur VKA. Stand Februar 2017. Frankfurt am Main. 
DuEPublico

Duisburg-Essen Publications online
UNIVERSITÄT

DE US'SER N R G

offen im Denken

$\mathbf{u b} \mid \begin{gathered}\text { universitäts } \\ \text { bibliothek }\end{gathered}$

Dieser Text wird über DuEPublico, dem Dokumenten- und Publikationsserver der Universität Duisburg-Essen, zur Verfügung gestellt. Die hier veröffentlichte Version der EPublikation kann von einer eventuell ebenfalls veröffentlichten Verlagsversion abweichen.

DOI: $\quad 10.3224 /$ indbez.v24i4.04

URN: urn:nbn:de:hbz:464-20200702-164847-2 